\title{
Nonsurgical rhinoplasty using soft tissue fillers
}

\author{
Yuyang Chu', Jonathan Bacos ${ }^{2}$, Sasha Becker ${ }^{1}$ \\ 'Northwestern University, Feinberg School of Medicine, Chicago, IL 60611, USA \\ ${ }^{2}$ Department of Plastic Surgery, Medical College of Wisconsin, Milwaukee, WI 53226, USA.
}

Correspondence to: Mr. Yuyang Chu, Northwestern University, Feinberg School of Medicine, 420 East Superior Street, Chicago, IL 60611, USA. E-mail: yuyang.chu@northestern.edu

How to cite this article: Chu Y, Bacos J, Becker S. Nonsurgical rhinoplasty using soft tissue fillers. Plast Aesthet Res 2020;7:73. http://dx.doi.org/10.20517/2347-9264.2020.169

Received: 24 Aug 2020 First Decision: 12 Nov 2020 Revised: 16 Nov 2020 Accepted: 24 Nov 2020 Published: 10 Dec 2020

Academic Editor: Wen-Guo Cui Copy Editor: Cai-Hong Wang Production Editor: Jing Yu

\begin{abstract}
Nonsurgical rhinoplasty, also known as liquid rhinoplasty, is a filler-based approach to treating deformities of the nose. Despite the potential for serious complications such as tissue necrosis and blindness, patients' desires for rapid results with minimal downtime and low costs have served as an impetus for rhinoplasty surgeons to become skilled injectors. Additionally, many physicians that are less skilled in rhinoplasty may be emboldened to perform a simpler procedure. While soft tissue filler is not always a viable alternative to rhinoplasty, it can be a useful adjunct or stand-alone treatment for managing a drooping nasal tip, minor asymmetries, or a dorsal hump. This article provides an overview of liquid rhinoplasty and how to best obtain the patient's desired aesthetic result.
\end{abstract}

Keywords: Nonsurgical rhinoplasty, hyaluronic acid, calcium hydroxyl apatite, injectables

\section{INTRODUCTION}

The application of soft tissue filler is the second most common minimally invasive cosmetic procedure reported in the 2018 plastic surgery statistics report ${ }^{[1]}$. Overall, fillers provide an enticing alternative to surgical procedures for patients seeking an aesthetic improvement. This is due to the minimal downtime, low cost, and low risk profile associated with fillers. While soft tissue fillers initially made their debut in the 1970s, it was not until the mid 1980s that physicians began reporting contouring noses with injectables ${ }^{[2,3]}$. Recently, the popularity of injectable fillers has increased significantly. Filler use rose $274 \%$ between 2000 and $2015^{[4]}$. Initial reports consisted of using bovine collagen and silicone, but a transition to more reliable and safe formulations of hyaluronic acid (HA) and calcium hydroxylapatite (CaHa) have allowed clinicians

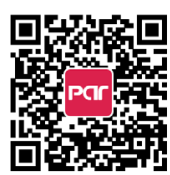


to obtain remarkable results with nonsurgical rhinoplasties. Nonetheless, the most important factor in providing optimal treatment is patient selection.

Nasal reshaping with HA filler is often most helpful as a post rhinoplasty adjunct. However, many authors have reported the use of HA filler in an unoperated nose. When treating the virgin nose, injectors must be cognizant that the use of large amounts of HA filler to correct a deformity may lead to vascular compromise, thus making liquid rhinoplasty a poor solution for significant nasal deformities. The most appropriate candidates for nonsurgical rhinoplasty are often those with mild cosmetic defects. Patients who tend to show the best results are those with a mildly deviated nose, mild dorsal hump, slight imbalances from previous surgery, and high nasal tip with flat radix ${ }^{[5,6]}$. Following a physical examination, the clinician must determine whether a reasonable improvement in the defect can be achieved, and inform the patient of alternatives such as surgical rhinoplasty as well as risks and benefits in order to improve patient understanding and set reasonable expectations.

For some patients, there is a contraindication to using fillers. This group of patients includes individuals with a history of bleeding disorders, autoimmune disorders, and hypersensitivity to filler composition. Patients who are pregnant or are breastfeeding should avoid filler injections. In addition, patients who show signs of inflammation or infection near injection sites should not receive the procedure. Extreme caution should be taken with patients that have undergone a recent surgical rhinoplasty. Additionally, all patients are advised to avoid substances that impair hemostasis for one week to prevent bruising and bleeding ${ }^{[6]}$. Further, it is suggested that patients with suspected body dysmorphic disorder (BDD) see a psychiatrist before undergoing liquid rhinoplasty. This is done in an effort to avoid patient dissatisfaction, as there is an increased occurrence of BDD in people seeking a rhinoplasty ${ }^{[7]}$.

Successful application of filler also relies on choosing the appropriate product. Permanent fillers are generally not used due to their irreversible nature and the risk of granuloma formation. Currently, HA filler is the most common filler used in liquid rhinoplasty, with up to $80 \%$ of liquid rhinoplasty procedures using $\mathrm{HA}^{[6]}$. Many clinicians prefer HA because it is soft, provides a natural feel and can be reversed and quickly dissolved with hyaluronidase ${ }^{[8]}$. However, some clinicians opt for CaHa. While CaHa cannot be easily reversed, it provides a longer lasting effect and is more durable over time. CaHa fillers usually last between 12 and 18 months, while HA fillers last between 6 and 12 months ${ }^{[9]}$. Therefore, HA fillers may require more repeated injections in order to maintain the desired form or shape. However, while CaHa requires less product, more stiffness can cause increased discomfort after the procedure ${ }^{[10]}$.

\section{TECHNIQUE}

Prior to injection, topical anesthetic ointment is applied 15-30 min before the procedure.

Fillers can be reconstituted with the addition of $1 \%$ lidocaine with epinephrine $(1: 100,000)$ to reduce discomfort, viscosity, and ultimately result in less postprocedural ecchymosis. As the average injection volume for nasal reshaping is $0.4 \mathrm{~mL}$ of $\mathrm{HA}$ per injection site, addition of $0.004 \mathrm{~mL}$ of lidocaine is suggested $^{[11]}$.

Injections can be delivered with a needle or cannula. Previously, it has been shown that cannulas provide improved patient comfort, with decreased side effects such as edema and ecchymosis, especially in highly vascular regions $^{[12]}$. Additionally, Pavicic et al ${ }^{[12]}$ demonstrated that filler placement with cannulas provides improved precision. Nonetheless, injection technique is highly variable and often based on prior training and comfort ${ }^{[13]}$. Due to the reduced risk of vascular complications and bruising, the use of microcannulas has become more popular [Figures 1 and 2]. 


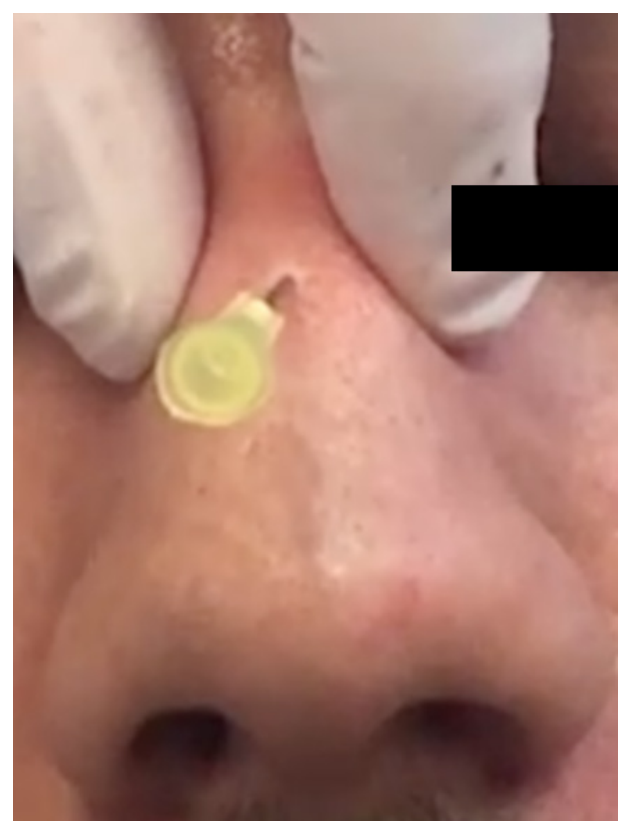

Figure 1. Injection site is created with a 23-gauge needle

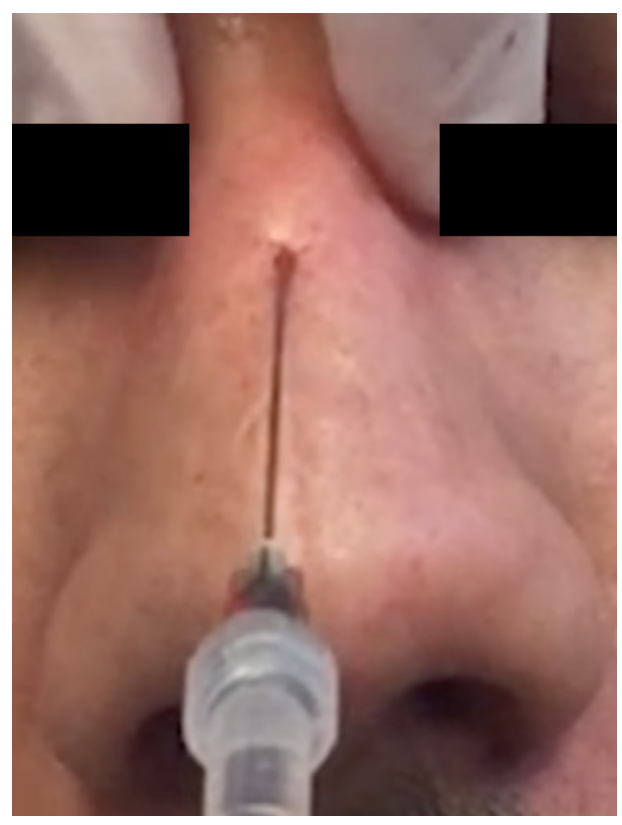

Figure 2. 22-gauge microcannula insertion through the injection site

Understanding the anatomy of the nose is critical to minimizing the risk of complications and optimizing the aesthetic outcome. From superficial to deep, the four layers that comprise the nose are the superficial fatty layer, the SMAS (superficial musculo-aponeurotic system), the deep fatty layer, and periosteum ${ }^{[14]}$. The superficial fatty and SMAS layers contain major blood vessels of the nose. To minimize and avoid vascular occlusion, skin necrosis, and blindness, filler injections should be delivered into the deep fatty layer, which is between the SMAS and the periosteum. In regards to the dorsal nasal artery, it is imperative that the injector verifies that the tip of the cannula is in the preperiosteal plane. This helps to avoid accidental arterial cannulation which can often lead to serious ocular complications ${ }^{[15]}$. 


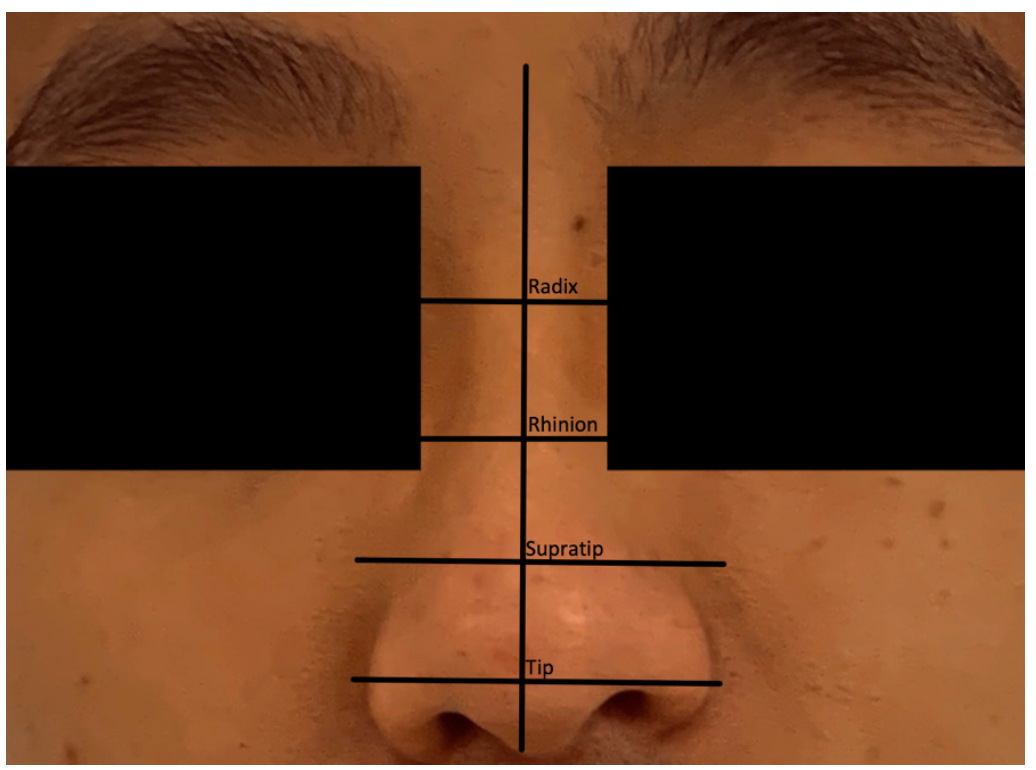

Figure 3. Anatomic injection sites

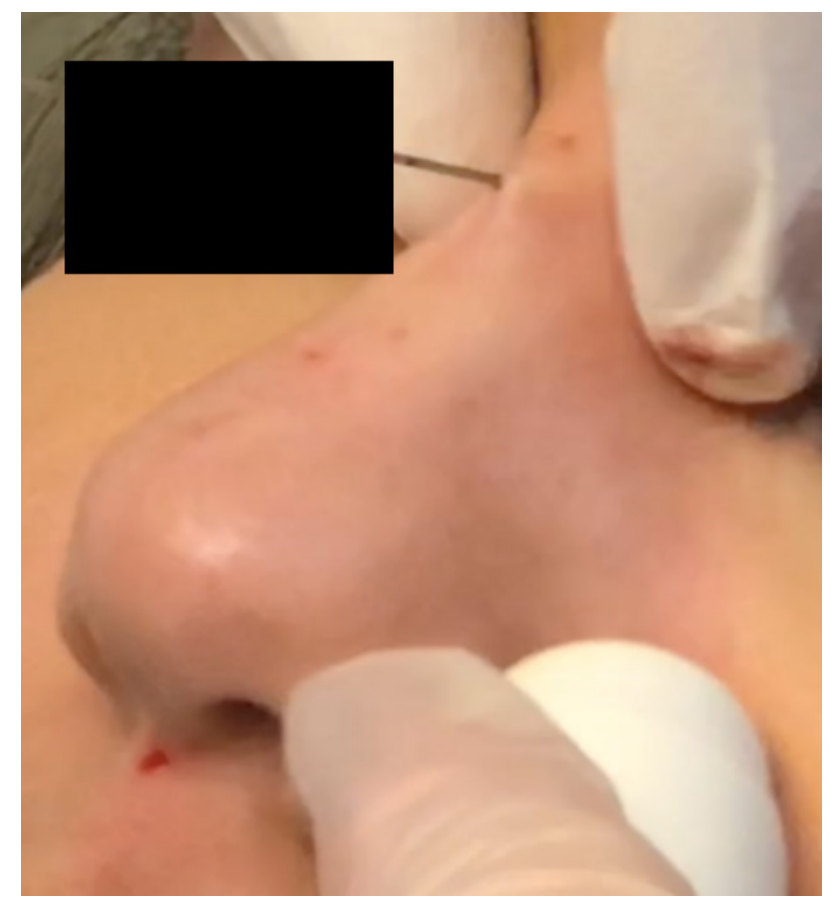

Figure 4. Initial treatment of the radix

Additionally, from superior to inferior, the nose can be broken down into four segments starting with the radix, followed by the rhinion, supratip and tip [Figure 3]. A top down approach is often preferred to address the nose [Figures 4 and 5].

Patients may also benefit from improved nasal tip definition and may stand to benefit from enhancement of the nasolabial angle. Nasal tip contouring can involve injections into the alar margin, nasal spine, dome area, and columellar space ${ }^{[16]}$. Injecting into the alar margin can help correct alar retraction. Injecting into the nasal spine elevates the nasal tip, which can help contribute to a more obtuse nasolabial angle ${ }^{[17]}$. Filling 


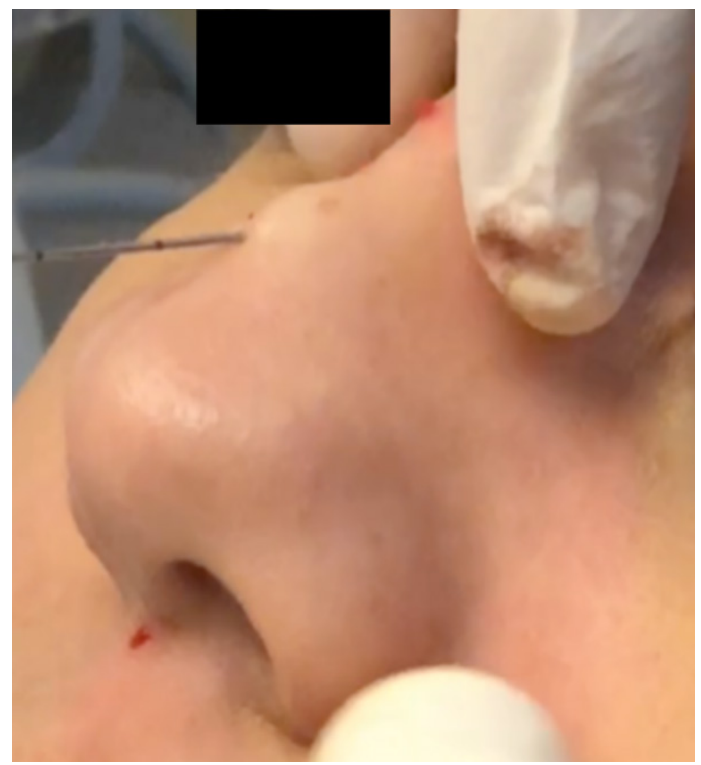

Figure 5. Treatment of the rhinion following treatment of the radix

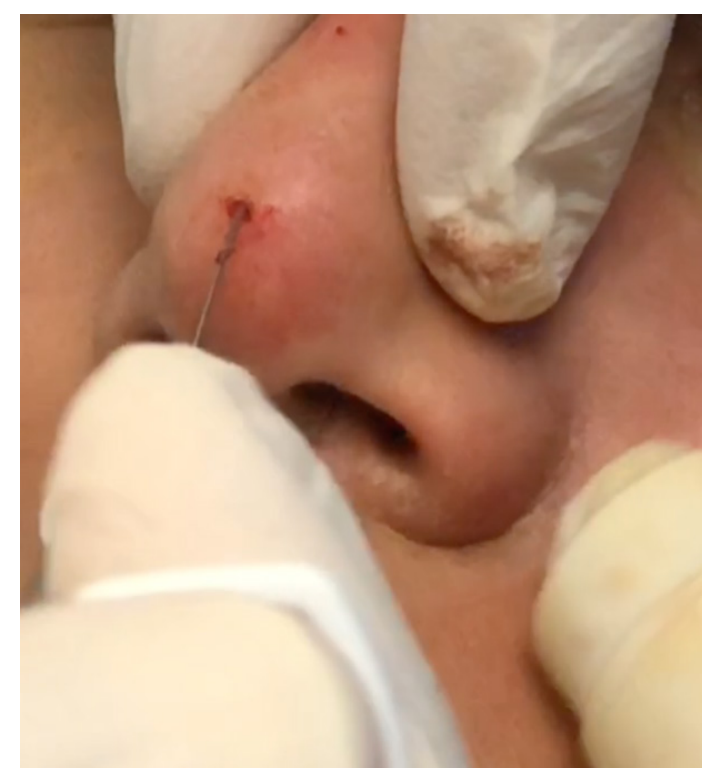

Figure 6. Treatment of the nasal tip

the columellar space can help correct columellar retraction by providing additional support for the nasal tip. Many injectors prefer injection of the tip prior to treatment of the supratip because the tip has relatively weaker supporting structures, which makes it difficult to ascertain the final amount of projection that can be achieved at the desired location [Figure 6].

Overall, general safe injection technique involves injecting below the SMAS plane into the deep fatty layer and staying midline whenever possible to decrease the likelihood of asymmetries. Consider the use of a blunt cannula to decrease the chance of vascular complications. When injecting, a two-hand approach is recommended: one hand is responsible for the action of injecting while the other hand helps to stabilize and guide the needle into the skin. After injection, light massaging of the area is recommended to help form a smooth contour. Following the above technique can help achieve safe, reproducible results. 


\section{Novel techniques}

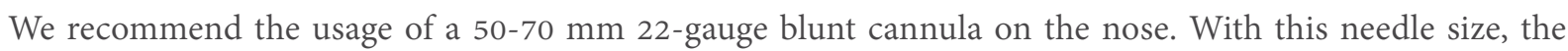
nose can be treated through one port, reducing the number of additional needle sticks. The large size of the needle also decreases injection pressure and the risk of penetrating a blood vessel, and because the nose is a highly vascular region, this increases the safety of the procedure. We also recommend blood aspiration before injection to diminish the risk of vascular complications. A 2015 article reports that the aspiration was reliable in $53 \%$ of cases in predicting accidental entry into a vessel ${ }^{[18]}$. When augmenting the nasal dorsum with HA filler injections, we suggest compressing vessels against the nose bilaterally with one hand, while holding the cannula in the other hand and injecting up and down the nose. These injections can also lift the nasal tip and the upper lip. If also correcting for ala depression, ports can be inserted bilaterally to the nasal tip to augment the depression.

Moreover, while we recommend the use of HA, it is of note that Radiesse injections have been increasing in popularity in recent years. Radiesse injections contain calcium hydroxylapatite and are preferred by some physicians due to specific properties of calcium hydroxylapatite, including its long duration of hold, moldability, high viscosity, high elasticity, and low immunogenicity ${ }^{[19]}$. Along with the changing materials of liquid rhinoplasty, the applications of liquid rhinoplasty have been expanding to become a corrective treatment post-surgical rhinoplasty. A 2016 study found that 27\% of patients undergoing liquid rhinoplasty chose to as a secondary correction for surgical rhinoplasty ${ }^{[20]}$.

Finally, it is also critical to acknowledge that there is a significant gap in data on filler injections in Chinese women of the Han nationality, despite liquid rhinoplasty being popular among this patient population. Technique can be highly modified to match patient preference and need. In a 2015 study of 280 Chinese women of Han nationality, a 5-step process using a blunt and sharp needle was found to be most effective ${ }^{[21]}$. The first step includes using a 26-gauge sharp needle to inject HA into the periosteum of the nasal spine in order to augment the nasolabial angle. The second step is to use the 26-gauge needle to create an injection site on the nasal tip subcutaneous to the nasal process. Filling should then be performed several times (3-4) in order to lengthen the columella. Third, nasal tip filling is completed using 0.1-0.2 mL of HA with the 26-gauge needle. However, it is suggested to do a bilateral injection rather than a midline injection in this patient population as it achieves a more natural aesthetic appearance. The next step, while injecting the nasal dorsum, is to make several injections of 0.2-0.6 mL of HA starting at the nasal root and ending at the nasal tip. This is done first in the supraperiosteal and supraperichondrial layers and then in the intramuscular and subcutaneous regions. The fifth and final step is to use a 26-gauge needle on the inner part of the eyebrow to enhance the nasal root appearance ${ }^{[21]}$. Of note, a 23 -guage needle can be used for any of the above steps if there is concern for vascular complications.

\section{COMPLICATIONS}

Complications of liquid rhinoplasty can be divided into two categories: early and delayed onset. Early onset complications are characterized by an appearance after several hours, while delayed onset complications are characterized by an appearance after weeks or years. The most common early complication is asymmetry, which can be prevented by staying as close to the midline as possible. There can also be hypersensitivity reactions to the filler content leading to fever, pruritis, and pain. Surface nodules and unevenness can also occur along with erythema, edema, ecchymosis, and pain at the sites of the injection ${ }^{[22]}$. Another early complication is the Tyndall effect, which occurs due to superficial filling and is characterized by a bluish tint on the skin. Delayed complications include granuloma formation, scarring, and skin color change.

Some of the more serious complications are infection, which has a complication rate of $0.04 \%$ and skin necrosis, which has a complication rate of $0.06 \%^{[23]}$. Skin necrosis is caused by vascular occlusion, which can be a result of direct intravascular injection, or from a compressive effect on local vessels. The main 
arteries at risk of vascular complications in the nose are the lateral nasal artery, which predominately supplies the nasal tip, and the dorsal nasal artery, which supplies the upper dorsum of the nose. There are several anastomoses in the nasal region between the external and internal carotid systems, whose blood flow can be reversed with injections, creating a potential retrograde embolization and subsequent stroke or blindness ${ }^{[24]}$. To diminish the risk of vascular complications, injectors should aspirate before injection, inject slowly with minimal pressure, use products with vasoconstrictors, and use blunt, large microcannulas in the avascular deep plane below the level of the SMAS ${ }^{[25]}$.

In addition to complications and safety concerns, novel research has shown that liquid rhinoplasty can have an insufficient effect in some patient populations. A 2016 study notes that $10 \%$ of 250 patients reported that the filler injection lasted only a short duration of less than 6 months or did not achieve the desired aesthetic result ${ }^{[20]}$. This requires patients to receive repeat injections, which increases the risk of complications and may cause potential financial burden.

\section{Novel treatments of complications}

Recent literature highlights the treatment for the complications explained above. Immediate adverse effects of HA, such as ischemia, can be corrected in a variety of ways. Hyaluronidase is the standard treatment, and there are several supplemental treatments available to help spread the hyaluronidase effectively. These supplemental treatments include topical nitropaste, oral acetylsalicylic acid, warm compresses, and massage. Nitropaste and warm compresses promote vasodilation, which promotes spread of the hyaluronidase through tissues. The pressure caused by massage helps to distribute the hyaluronidase rapidly, and acetylsalicylic acid provides an anti-clotting effect ${ }^{[2]}$.

Additionally, a 2019 study elucidates a procedural strategy to reduce complications. In the study, researchers injected a colored filler into the dorsum of a cadaver. In three of six cadavers, the filler was injected into the superficial layer and in the other three cadavers, the filler was injected by direct percutaneous injection into the deep, avascular layer. Injection through the glabella into the deep layer allowed for more accurate injection. This can decrease the chance of vascular injury, which can reduce the risk of necrosis and vision $\operatorname{loss}^{[27]}$.

\section{CONCLUSION}

Liquid rhinoplasty with HA fillers is a safe procedure with positive aesthetic results when performed by a trained professional. Post procedure, many patients will have self-limiting side effect such as mild erythema and swelling. Very rarely, some patients will suffer infections and local tissue necrosis. Using correct injection technique such as filling only in the deep fatty later and staying in the midline can help reduce the chance of adverse effects. Further, having a targeted plan on the location and order of injections can help achieve the desired aesthetic result with safety, consistency, and reproducibility.

\section{DECLARATIONS}

\section{Authors' contributions}

All contributed equally to the research, writing, and editing of the manuscript: Chu Y, Bacos J, Becker S

\section{Availability of data and materials}

Not applicable.

\section{Financial support and sponsorship}

None. 


\section{Conflict of interest}

All authors declared that there are no conflicts of interest.

\section{Ethical approval and consent to participate}

Not applicable.

\section{Consent for publication}

Not applicable.

\section{Copyright}

(c) The Author(s) 2020.

\section{REFERENCES}

1. American Society of Plastic Surgeons Release. 2018 Plastic surgery statistics report. Available from: https://www.plasticsurgery.org/ documents/News/Statistics/2018/plastic-surgery-statistics-full-report-2018.pdf. [Last accessed on 24 Jun 2020]

2. Knapp TR, Vistnes LM. The augmentation of soft tissue with injectable collagen. Clin Plast Surg 1985;12:221-5.

3. Webster RC, Hamdan US, Gaunt JM, Fuleihan NS, Smith RC. Rhinoplastic revisions with injectable silicone. Arch Otolaryngol Head Neck Surg 1986;112:269-76.

4. Wang LL, Friedman O. Update on injectables in the nose. Curr Opin Otolaryngol Head Neck Surg 2017;25:307-13.

5. Kontis TC. Nonsurgical rhinoplasty. JAMA Facial Plast Surg 2017;19:430-1.

6. Raggio BS, Asaria J. Filler rhinoplasty. In: StatPearls. Treasure Island (FL): StatPearls Publishing; 2020.

7. Sarwer DB, Wadden TA, Pertschuk MJ, Whitaker LA. Body image dissatisfaction and body dysmorphic disorder in 100 cosmetic surgery patients. Plast Reconstr Surg 1998;101:1644-9.

8. Betemps JB, Marchetti F, Lim T, et al. Projection capacity assessment of hyaluronic acid fillers. Plast Aesthet Res 2018;5:19.

9. Sundaram H, Voigts B, Beer K, Meland M. Comparison of the rheological properties of viscosity and elasticity in two categories of soft tissue fillers: calcium hydroxylapatite and hyaluronic acid. Dermatol Surg 2010;36 Suppl 3:1859-65.

10. Kablik J, Monheit GD, Yu L, Chang G, Gershkovich J. Comparative physical properties of hyaluronic acid dermal fillers. Dermatol Surg 2009;35 Suppl 1:302-12.

11. Hedén P. Nasal reshaping with hyaluronic acid: an alternative or complement to surgery. Plast Reconstr Surg Glob Open 2016;4:e1120.

12. Pavicic T, Frank K, Erlbacher K, et al. Precision in dermal filling: a comparison between needle and cannula when using soft tissue fillers. J Drugs Dermatol 2017;16:866-72.

13. Bacos JT, Dayan SH. Superficial dermal fillers with hyaluronic acid. Facial Plast Surg 2019;35:219-23.

14. Moon HJ. Injection rhinoplasty using filler. Facial Plast Surg Clin North Am 2018;26:323-30.

15. Tansatit T, Apinuntrum P, Phetudom T. Facing the worst risk: confronting the dorsal nasal artery, implication for non-surgical procedures of nasal augmentation. Aesthetic Plast Surg 2017;41:191-8.

16. Daniel RK, Letourneau A. Rhinoplasty: nasal anatomy. Ann Plast Surg 1988;20:5-13.

17. Menick FJ. Anatomic reconstruction of the nasal tip cartilages in secondary and reconstructive rhinoplasty. Plast Reconstr Surg 1999;104:2187-98; discussion 2199-201.

18. Casabona G. Blood aspiration test for cosmetic fillers to prevent accidental intravascular injection in the face. Dermatol Surg 2015;41:841-7.

19. Rho NK, Chang YY, Chao YY, et al. Consensus recommendations for optimal augmentation of the asian face with hyaluronic acid and calcium hydroxylapatite fillers. Plast Reconstr Surg 2015;136:940-56.

20. Rosenberger ES, Toriumi DM. Controversies in revision rhinoplasty. Facial Plast Surg Clin North Am 2016;24:337-45.

21. Han X, Hu J, Cheng L, Li F. Multiplane hyaluronic acid (EME) in female Chinese rhinoplasty using blunt and sharp needle technique. $J$ Plast Reconstr Aesthet Surg 2015;68:1504-9.

22. Dayan SH, Arkins JP, Brindise R. Soft tissue fillers and biofilms. Facial Plast Surg 2011;27:23-8.

23. Harb A, Brewster CT. The nonsurgical rhinoplasty: a retrospective review of 5000 treatments. Plast Reconstr Surg 2020;145:661-7.

24. Kim DW, Yoon ES, Ji YH, Park SH, Lee BI, Dhong ES. Vascular complications of hyaluronic acid fillers and the role of hyaluronidase in management. J Plast Reconstr Aesthet Surg 2011;64:1590-5.

25. Beleznay K, Carruthers JD, Humphrey S, Jones D. Avoiding and treating blindness from fillers: a review of the world literature. Dermatol Surg 2015;41:1097-117.

26. DeLorenzi C. Complications of injectable fillers, part 2: vascular complications. Aesthet Surg J 2014;34:584-600.

27. Jung GS, Chu SG, Lee JW, et al. A safer non-surgical filler augmentation rhinoplasty based on the anatomy of the nose. Aesthetic Plast Surg 2019;43:447-52. 